\title{
A survey of resistance to colchicine treatment for French patients with familial Mediterranean fever
}

\author{
Alice Corsia', Sophie Georgin-Lavialle², Véronique Hentgen ${ }^{3}$, Eric Hachulla ${ }^{4}$, Gilles Grateau², Albert Faye
} Pierre Quartier ${ }^{6}$, Linda Rossi-Semerano ${ }^{1}$ and Isabelle Koné-Paut ${ }^{*}$

\begin{abstract}
Background: Colchicine is the standard treatment for familial Mediterranean fever (FMF), preventing attacks and inflammatory complications. True resistance is rare and yet not clearly defined. We evaluated physicians' definition of colchicine resistance and report how they manage it.

Patients and methods: We recruited patients with a clinical diagnosis of FMF, one exon-10 Mediterranean fever (MEFV) gene mutation and considered resistant to colchicine, via networks of expert physicians. Clinical, biological characteristics and information about colchicine treatment (dose adjustment, compliance) were collected. The severity of FMF was assessed by the Tel Hashomer criteria.

Results: We included 51 patients, most females (55\%), mean age $34 \pm 23.1$ years years (range 4.7-86.3). Overall, 58\% (27/47) patients had homozygous M694 MEFV gene mutations. Seventeen of 42 patients (40\%) declared full adherence to colchicine treatment, greater for children (48\%) than adults (22\%). Physicians considered colchicine resistance with > 6 attacks/year $(n=21 / 51,42 \%),>4$ attacks in the last 6 months $(n=13 / 51,26 \%)$, persistent inflammation $(n=23 / 51,45 \%)$, renal amyloidosis in $(n=6 / 28,22 \%)$ of adult patients and intolerance to an increase in colchicine dose $(n=10 / 51,19 \%)$, and other reasons $(n=13 / 51,23 \%)$, including chronic arthralgia $(n=6 / 51,12 \%)$. Interleukin 1-targeting drugs represented the only alternative treatments in addition to daily colchicine.

Conclusion: Resistance to colchicine is rare (<10\% of patients) and mostly observed in severe MEFV genotypes. The main reasons for physicians assessing resistance were severe clinical symptoms, persistent subclinical inflammation, and secondary amyloidosis. Low adherence to colchicine treatment is a key component of resistance.
\end{abstract}

Keywords: Familial Mediterranean fever, Colchicine, Resistance to treatment, Compliance, Anti interleukin 1

\section{Significance and innovations}

- In pediatric care setting, the most important reason to consider resistance to colchicine treatment in patients with FMF was a high frequency of attacks.

- In adult care setting, the most important reason to consider resistance to colchicine treatment was secondary amyloidosis.

\footnotetext{
*Correspondence: Isabelle.kone-paut@aphp.fr

'Department of Paediatrics Rheumatology, CEREMAl, Bicêtre Hospital, AP-HP,

University of Paris SUD, Le Kremlin-Bicêtre, France

Full list of author information is available at the end of the article

- In both group, digestive intolerance, persistent subclinical inflammation and joint symptoms contributed to colchicine resistance.

- Overall full compliance to colchicine treatment was low (40\%), especially in the adult care setting group $(22 \%)$

\section{Background}

Familial Mediterranean fever (FMF) is the historical prototype of a group of inherited inflammatory disorders of innate immunity, so-called autoinflammatory diseases. FMF is essentially observed in Mediterranean populations, affecting more than 100,000 people [1]. The main clinical characteristics are self-limited acute febrile attacks accompanied 
by peritoneum, pleura, skin, muscle and joint inflammation. FMF severely impairs quality of life and causes secondary inflammatory complications such as amyloid A amyloidosis [2].

Daily treatment with colchicine was introduced in 1972 to prevent FMF attacks and secondary amyloidosis by also reducing the level of sub-clinical inflammation [2]. The action mechanisms of colchicine are diverse and still unclear, but its ability to disrupt the cytoskeleton probably plays an important part. Colchicine may have anti-inflammatory effects in FMF by reorganizing the actin cytoskeleton and down regulating Mediterranean fever $(M E F V)$ gene expression. To date, a median dose of 1-2 mg daily of colchicine remains the mainstay of FMF treatment, allowing for significant reduction or absence of acute attacks in more than $90 \%$ of cases $[3,4]$.

Although considered generally safe and effective, daily colchicine treatment for FMF has some limitations. Indeed, colchicine has a narrow therapeutic window at blood levels $<7 \mathrm{ng} / \mathrm{mL}$, but at doses $>10 \mathrm{ng} / \mathrm{mL}$, it has serious toxic effects and can lead to potentially fatal outcomes. This peculiarity is also associated with digestive intolerance, which limits the possibility of increasing the daily dose to obtain full therapeutic effect in patients with the most severe (inflammatory) phenotypes. In daily practice, although colchicine remains an inexpensive and effective means to control FMF inflammation, 5 to $10 \%$ of patients will not be able to achieve complete response, which raises the possibility of new therapeutic approaches such as interleukin 1 (IL-1)-targeting drugs [5-7].

Considering the very high cost of these treatments for FMF, we aimed to survey when and how adult and paediatric physicians, consider resistance to colchicine in patients with FMF and to report how they handle this situation in their practice.

\section{Patients and methods Patients and setting}

We retrospectively reviewed charts of patients identified through reference centres and networks of inflammatorydisease expert physicians. Electronic mailing lists of French paediatric and adult rheumatologist societies were used to request medical histories of FMF patients considered resistant to colchicine treatment. We asked physicians to report patients with a clinical diagnosis of FMF and at least one pathogenic $M E F V$ mutation whom they considered resistant to colchicine. A dedicated questionnaire was used to collect data on demographics (age, sex, ethnic origin), $M E F V$ mutation type, age at first symptoms and at diagnosis, description of clinical symptoms before and under colchicine treatment, biological inflammatory markers tested during and between attack periods before and during colchicine treatment, associated inflammatory diseases, tolerance to treatment, dose adjustments, and evaluation of adherence to treatment. Disease severity was assessed by the Tel Hashomer criteria [8]. Finally, we analysed the reasons for physicians considering their patients resistant to treatment and collected the alternative attitudes and treatments used. We excluded patients with concomitant diseases and manifestations that might mimic FMF, such as spondyloarthropathies or Crohn's disease, to avoid confusion in evaluating disease severity.

\section{Statistical analysis}

Because we had both paediatric and adult care setting populations, we first divided the patients into these two subgroups, with the paediatric care population ranging in age from 0 to 21 years. We chose this age limit because several patients were still seeing paediatricians from age 18 to 21 years. General statistics are reported as mean \pm SD. All descriptive results are given with $95 \%$ confidence intervals (95\% CIs). Analyses involved the chi-square test for categorical variables and $t$-test for continuous variables. Microsoft Excel vXI was used for analysis. $P<0.05$ was considered statistically significant.

\section{Results \\ Study patients}

We recruited 51 patients with a clinical diagnosis of FMF from nine centres, in which four were paediatric departments. Mean age was $34 \pm 23.1$ years (range $4.7-$ 86.3 ), with 23 males (45\%); 23 patients (45\%) under 21 years old were in paediatric care setting, in whom 3 between 18 and 21 years old. Thirty (59\%) were Sephardic Jews, seven (14\%) were from Turkey or Armenia, ten (20\%) were from North Africa, two (4\%) were from Lebanon and two (4\%) were of mixed ethnic background. The mean age at disease onset was $7.8 \pm 8.7$ years (range 1 month to 40 years); 17 (33\%) had disease onset $\leq 2$ years. All patients with available data $(n=47 / 51$, 93\%) carried pathogenic mutations in exon 10 of $M E F V$ except one girl and a woman with a complex allele including $M E F V$ deletion. (Table 1).

\section{Frequency and duration of attacks before and after colchicine treatment}

We had data for 36 patients (72\%) before colchicine treatment; $14(50 \%)$ patients in adult care setting (group I), 22 (96\%) patients in pediatric care setting (group II), (Table 1). The frequency of attacks was significantly higher in patients of group II than in those of group I $(p=0.019)$. The mean duration of attacks in group II was $50 \mathrm{~h}$ (range 12-84), which was significantly lower than in group I: $67 \mathrm{~h}$ (range 36-96; $p=0.025$ ). Under colchicine treatment, for 27 (53\%) patients, the number of attacks was $>1$ /month $(15(65 \%)$ in group II vs. $12(43 \%)$ in group I; $p=0.27)$. These data were unavailable for $28 \%$ of patients ( $4 \%$ in group II vs. 
Table 1 Clinical and demographic characteristics of 51 patients with familial Mediterranean fever before colchicine treatment

\begin{tabular}{|c|c|c|c|c|}
\hline & $\begin{array}{l}\text { All patients } \\
n=51\end{array}$ & $\begin{array}{l}\text { Group } I^{a} \\
n=28\end{array}$ & $\begin{array}{l}\text { Group } \|^{b} \\
n=23\end{array}$ & $p$ values \\
\hline Age at diagnosis (years) & $7.8 \pm 8.7$ & $12.2 \pm 9.6$ & $2.7 \pm 2.8$ & 0.001 \\
\hline Sex & & & & 0.166 \\
\hline Male & $23(45 \%)$ & $10(36 \%)$ & $13(57 \%)$ & \\
\hline Female & $28(55 \%)$ & $18(64 \%)$ & $10(43 \%)$ & \\
\hline MEFV mutation & & & & 0.235 \\
\hline M694V/M694V & $27(51 \%)$ & $13(43 \%)$ & $14(61 \%)$ & \\
\hline M694V/M694I & $7(13 \%)$ & $4(13 \%)$ & $3(13 \%)$ & \\
\hline M694I/M694I & $1(2 \%)$ & $0(0 \%)$ & $1(4 \%)$ & \\
\hline M680I/M680I & $1(2 \%)$ & $0(0 \%)$ & $1(4 \%)$ & \\
\hline M694VN726A & $1(2 \%)$ & $1(3 \%)$ & $0(0 \%)$ & \\
\hline M694V/I591T & $1(2 \%)$ & $1(3 \%)$ & $0(0 \%)$ & \\
\hline M694V/E148Q & $1(2 \%)$ & $1(3 \%)$ & $0(0 \%)$ & \\
\hline M726V/M680V & $1(2 \%)$ & $1(3 \%)$ & $0(0 \%)$ & \\
\hline M694V/TRAPS polymorph. & $1(2 \%)$ & $0(0 \%)$ & $1(4 \%)$ & \\
\hline E148Q/1692delN726A & $1(2 \%)$ & $0(0 \%)$ & $1(4 \%)$ & \\
\hline M694V/- & $4(8 \%)$ & $1(3 \%)$ & $3(13 \%)$ & \\
\hline M694I/- & $1(2 \%)$ & $1(3 \%)$ & $0(0 \%)$ & \\
\hline NA & $4(7.5 \%)$ & $4(13 \%)$ & $0(0 \%)$ & \\
\hline Frequency of attacks & & & & 0.019 \\
\hline$>1 / 2$ weeks & $13(26 \%)$ & $5(18 \%)$ & $8(35 \%)$ & \\
\hline$>1 /$ month & $17(33 \%)$ & $7(25 \%)$ & $10(44 \%)$ & \\
\hline$>1 / 3$ months, $<1 /$ month & $3(6 \%)$ & $1(4 \%)$ & $2(9 \%)$ & \\
\hline$<1 / 3$ months, $>3 /$ year & $2(4 \%)$ & $1(4 \%)$ & $1(4 \%)$ & \\
\hline$<3$ /year & $1(2 \%)$ & $0(0 \%)$ & $1(4 \%)$ & \\
\hline NA & $15(29 \%)$ & $14(50 \%)$ & $1(4 \%)$ & \\
\hline Duration of attacks (hr) & $57 \pm 22.4$ & $67 \pm 17$ & $50 \pm 24$ & 0.025 \\
\hline NA & 17 (33\%) & $13(46 \%)$ & $4(17 \%)$ & \\
\hline Delay to treatment (years) & $8.7 \pm 12.9$ & $15 \pm 15.9$ & $2.4 \pm 2.6$ & $<0.001$ \\
\hline Total & 51 & $28(55 \%)$ & $23(45 \%)$ & \\
\hline
\end{tabular}

Data are $\mathrm{n}(\%)$ or mean \pm SD

${ }^{a}$ Group I: Adult care setting

${ }^{\mathrm{b}}$ Group II: Pediatric care setting

$47 \%$ in group I). The mean duration of attacks under colchicine treatment was $55 \mathrm{~h}$ (range 12-120): $50.5 \mathrm{~h}$ (range 24-120) in group II vs. $60 \mathrm{~h}$ (range 11-96) in group I $(p=0.29)$.

\section{Severity of attacks before and during colchicine treatment}

We had data before colchicine treatment for $22(43 \%)$ patients for subjective evaluation, because the Tel Hashomer criteria include the response to colchicine. For all patients, the attack severity was $>4 / 10$ and for $13(59 \%)$, it was $>7$ / 10. During colchicine treatment, all 51 patients were classified by disease severity: disease was severe for $41 \%(n=21)$, intermediate for $35 \%(n=18)$, and mild for $23 \%(n=12)$.
Clinical features before and during colchicine treatment

Before Colchicine treatment, 44 patients (83\%) had fever during attack periods; these data were unavailable for 3 patients in group I (10\%). Overall, 33 patients (73\%) had abdominal pain: $91 \%$ in group II versus $57 \%$ in group I $(p=0.009)$. During Colchicine treatment, $10(22 \%)$ of patients had myalgia during attack periods, $2(8 \%)$ in group I vs $8(36 \%)$ in group II, $(p=0.21)$. Overall symptoms during attacks before and under colchicine treatment are detailed in Tables 2 and 3.

\section{Treatment responses and adherence}

The mean delay from the first attacks to colchicine treatment was $8.7 \pm 12.9$ years (range $0-57$ ): $2.4 \pm$ 2.6 years (range $0-9.8$ ) for group II versus $15 \pm 15.9$ years (range 0-57) for group I $(p=0.001)$. The mean maximal dose of colchicine given was $2.1 \pm 0.7$ for group I versus $1.8 \pm 0.5 \mathrm{mg}$ (equivalent $0.1 \pm 0.01 \mathrm{mg} / \mathrm{kg}$ ) for group II $(p=0.006)$. In all, $30 \%$ of group I patients had a dose $>$ $2 \mathrm{mg}$, with a maximal dose of $3 \mathrm{mg}$.

A total of 17 patients (32\%) had digestive intolerance [nine of group II (39\%) and 14 of group I (27\%)], with diarrhoea as a major symptom. One patient had severe muscle toxicity, not related to colchicine toxicity. Adherence to colchicine treatment was accurately evaluated in 42 patients (82\%): $73 \%$ of group I versus $96 \%$ of group II $(p=0.03)$. Overall, $40 \%$ of patients $(17 / 42)$ declared being fully adherent: $48 \%$ of group II versus $22 \%$ of group I patients $(p=0.08)$. Among these 42 patients, only three (7\%) had problem of intolerance to Colchicine.

Table 2 Patient's clinical symptoms before colchicine therapy

\begin{tabular}{lllll}
\hline & Population $n(\%)$ & $\begin{array}{l}\text { Group I } \\
n(\%)\end{array}$ & $\begin{array}{l}\text { Group II } \\
n(\%)\end{array}$ & $p$ values $^{*}$ \\
\hline Data available & $45(88 \%)$ & $22(79 \%)$ & $23(100 \%)$ & \\
Fever & $44(83 \%)$ & $21(70 \%)$ & $23(100 \%)$ & 0.3 \\
Abdominal pain & $33(73 \%)$ & $20(91 \%)$ & $13(57 \%)$ & 0.009 \\
Arthralgia & $21(41 \%)$ & $11(39 \%)$ & $10(44 \%)$ & 0.494 \\
Asthenia & $2(4 \%)$ & $1(4 \%)$ & $1(5 \%)$ & 0.974 \\
Myalgia & $8(18 \%)$ & $2(9 \%)$ & $6(26 \%)$ & 0.136 \\
Thoracic pain & $10(22 \%)$ & $6(27 \%)$ & $4(17 \%)$ & 0.4 \\
Diarrhoea & $2(4 \%)$ & $1(5 \%)$ & $1(4 \%)$ & 0.974 \\
Amyloidosis & $1(2 \%)$ & $1(5 \%)$ & $0(0 \%)$ & 0.301 \\
Skin rash & $15(33 \%)$ & $8(36 \%)$ & $7(30 \%)$ & 0.673 \\
Arthritis & $6(13 \%)$ & $4(18 \%)$ & $2(9 \%)$ & 0.349 \\
Peritonitis & $8(18 \%)$ & $2(9 \%)$ & $6(26 \%)$ & 0.14 \\
Vomiting & $2(4 \%)$ & $1(5 \%)$ & $1(4 \%)$ & 0.974 \\
Headache & $3(7 \%)$ & $0(0 \%)$ & $3(13 \%)$ & 0.08 \\
\hline
\end{tabular}

${ }^{¥}$ chi square test

${ }^{a}$ Group I: Adult care setting

${ }^{\mathrm{b}}$ Group II: Pediatric care setting 
Table 3 Patient's clinical symptoms under colchicine therapy

\begin{tabular}{lllll}
\hline Symptoms & Population $n(\%)$ & $\begin{array}{l}\text { Group I } \\
n(\%)\end{array}$ & $\begin{array}{l}\text { Group } I^{\mathrm{b}} \\
n(\%)\end{array}$ & $p$ value \\
\hline Data available & $46(90 \%)$ & $24(86 \%)$ & $22(96 \%)$ & \\
Fever & $40(87 \%)$ & $20(82 \%)$ & $20(91 \%)$ & 0.446 \\
Abdominal pain & $37(80 \%)$ & $19(79 \%)$ & $18(82 \%)$ & 0.821 \\
Arthralgia & $21(46 \%)$ & $11(46 \%)$ & $10(46 \%)$ & 0.979 \\
Asthenia & $13(28 \%)$ & $5(21 \%)$ & $8(36 \%)$ & 0.243 \\
Myalgia & $10(22 \%)$ & $2(8 \%)$ & $8(36 \%)$ & 0.021 \\
Thoracic pain & $9(20 \%)$ & $4(17 \%)$ & $5(23 \%)$ & 0.605 \\
Diarrhoea & $9(20 \%)$ & $2(8 \%)$ & $7(32 \%)$ & 0.045 \\
Amyloidosis & $9(20 \%)$ & $9(38 \%)$ & $0(0 \%)$ & 0.001 \\
Skin rash & $8(17 \%)$ & $2(8 \%)$ & $6(27 \%)$ & 0.090 \\
Arthritis & $7(15 \%)$ & $5(21 \%)$ & $2(9 \%)$ & 0.268 \\
Peritonitis & $6(13 \%)$ & $3(13 \%)$ & $3(14 \%)$ & 0.909 \\
Vomiting & $5(11 \%)$ & $4(17 \%)$ & $1(5 \%)$ & 0.187 \\
Headache & $5(11 \%)$ & $1(4 \%)$ & $4(18 \%)$ & 0.127 \\
\hline
\end{tabular}

${ }^{*}$ chi square test

${ }^{a}$ Group I: Adult care setting

${ }^{\mathrm{b}}$ Group II: Pediatric care setting

\section{Inflammatory markers under colchicine treatment}

C-reactive protein (CRP) and serum amyloid A protein (SAA) levels during attacks were evaluated in 30 (60\%) and 35 patients (69\%): mean values were $80 \pm 68$ and $327 \pm 304 \mathrm{mg} / \mathrm{L}$, respectively. Similarly, CRP and SAA levels between attacks were evaluated in 47 (92\%) and 35 patients (69\%): mean values were $34 \pm 44$ and $114 \pm$ $202 \mathrm{mg} / \mathrm{L}$, respectively. Levels did not differ between group I and group II populations (Fig. 1). Mean erythrocyte sedimentation rate between attacks was $34 \pm 25 \mathrm{mg} /$ L and was available for 28 patients (57\%; 83\% of group II vs. $32 \%$ of group I). Proteinuria between attacks was evaluated in 33 patients (66\%), with mean value $<0.1 \mathrm{~g} / \mathrm{L}$ in group II versus $0.7 \mathrm{~g} / \mathrm{L}$ in group I, seven patients known to have amyloidosis.

\section{Determination of inadequate response to colchicine (resistance) \\ Frequency of attacks}

Overall, $38 \%$ of patients kept a diary of their crises [70\% of group II vs $13 \%$ of group I $(p<0.01)]$; $42 \%$ were considered resistant to colchicine because of $>6$ attacks/year [ $65 \%$ of group II vs $23 \%$ of group I $(p=0.08)$ ].

\section{Amyloidosis and renal failure}

In group I, $22 \%$ patients were considered resistant because of renal amyloidosis under colchicine treatment $(p=0.02)$, and $20 \%$ because of renal failure, which was a contraindication to increase the dose to the optimum $(p=0.02)$ (Table 4$)$.

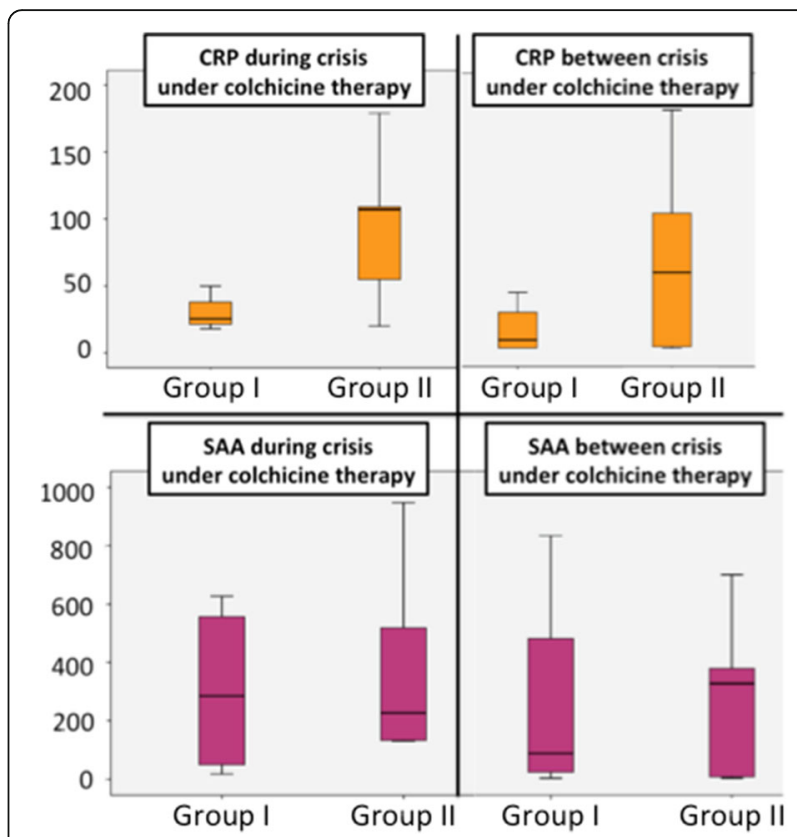

Fig. 1 C-reactive protein (CRP) level (mg/L) and serum amyloid A $(\mathrm{SAA})(\mathrm{mg} / \mathrm{L})$ levels before and under colchicine treatment. Horizontal lines are means; outer box edges are SD and whiskers are 95\% confidence intervals. *Group I: Adult care setting, **Group II: Pediatric care setting

\section{Other reasons}

In all, 23\% of patients had other reasons to be considered resistant to colchicine treatment: $50 \%$ had chronic arthralgia (three children $<18$ years in group II and three patients in group I).

All results are summarized in Table 4

\section{Concomitant and surrogate treatments}

Overall, $60 \%$ of patients (70\% of group II versus $53 \%$ of group I) used concomitant treatments to overcome FMF symptoms: non-steroidal anti-inflammatory drugs

Table 4 Determination of inadequate response to colchicine (resistance)

\begin{tabular}{|c|c|c|c|c|}
\hline Symptoms & $\begin{array}{l}\text { Population } \\
n(\%)\end{array}$ & $\begin{array}{l}\text { Group } 1^{a} \\
n(\%)\end{array}$ & $\begin{array}{l}\text { Group } \|^{\mathrm{b}} \\
n(\%)\end{array}$ & $p$ value $^{\ddagger}$ \\
\hline \multicolumn{5}{|l|}{ Frequency of attacks } \\
\hline > 6/year & $42 \%$ & $23 \%$ & $65 \%$ & $<0.08$ \\
\hline$>4 / 6$ months & $26 \%$ & $30 \%$ & $22 \%$ & ns \\
\hline Biological inflammation & $45 \%$ & $47 \%$ & $44 \%$ & ns \\
\hline Intolerance to treatment & $19 \%$ & $16 \%$ & $22 \%$ & ns \\
\hline Amyloidosis & & $22 \%$ & $0 \%$ & 0.02 \\
\hline Renal failure & & $20 \%$ & $0 \%$ & 0.02 \\
\hline Other reasons & $23 \%$ & $18 \%$ & $26 \%$ & ns \\
\hline
\end{tabular}

${ }^{¥}$ By chi-square test

*Group I: Adult care setting

${ }^{* *}$ Group II: Pediatric care setting 
(NSAIDs), steroids, or analgesics. Colchicine was still prescribed for $85 \%$ of patients, and $62 \%$ of these were treated receiving IL-1-targeted drugs (61\% in group II vs. $63 \%$ in group I). IL-1-targeted drugs were anakinra (79\%) or canakinumab (19\%), prescribed as continuous treatment for $83 \%$ of patients.

\section{Discussion}

To our knowledge, this is the first study evaluating unresponsiveness to colchicine treatment and physicians' assessment of it in a cohort of both adult and paediatric care patients with FMF living in a western European country. Resistance to colchicine is rare and mostly observed in severe $M E F V$ genotypes. Almost two-thirds of our 51 patients had homozygous M694 MEFV gene mutations. Less than half of evaluable patients declared full adherence to colchicine treatment, which was greater for children than adults. Physician's reasons for considering colchicine resistance included $>6$ attacks/year, $>4$ attacks in the last 6 months, and persistent inflammation. IL-1-targeting drugs represented the only alternative treatments in addition to daily colchicine. The main reasons for assessing resistance were severe clinical symptoms, persistent subclinical inflammation, and secondary amyloidosis. Low adherence to colchicine treatment is a key component of resistance, requiring appropriate patient education.

Renal failure is not a cause for resistance strictly speaking; nevertheless, renal failure impairs the possibility to increase the dose of colchicine. This is why we have considered it as a form of resistance with a cause. The cause of amyloidosis may be multifactorial, and not only related to non adherence or intolerance, but also due to true resistance or additional genetic and environmental factors.

The study was performed within a network of expert tertiary centres, which represents a major strength of the optimal care available in our country. The study gives important data because resistance to colchicine is responsible for increased disease-related morbidity, mortality and poor quality of life with FMF $[9,10]$. Thus, resistant patients seem to be good candidates for biologic treatment (i.e., anti-IL-1 treatments). Nevertheless, none of these drugs are being approved, and increasing their use may greatly increase the overall cost of care for FMF $[6,7,11]$.

Our study confirmed two important points: first, insufficient response to colchicine treatment is rare (about $10 \%$ of all patients seen in our centres); second, insufficient response affects mostly patients with the most severe disease pattern and pathogenic $M E F V$ mutations [10]. FMF severity in our patients was reflected by a high number of attacks per year, a high frequency of musculoskeletal involvement, and secondary amyloidosis. Of note, we excluded patients with amyloidosis as a presenting feature of FMF before colchicine treatment. Chronic musculoskeletal symptoms were another cause, well known to be generally benign and overcome with NSAIDs, but in a few cases, they can cause absenteeism from school or work. Some of these patients may show increased risk of developing secondary spondyloarthropathies, which was an exclusion criterion in our study [12]. Secondary amyloidosis appearing during the course of FMF was also a leading cause of resistance to colchicine, exclusively observed in the adult population. Physician's assessment of resistance to colchicine treatment was in accordance with the definition of the French Israeli consortium, "six or more typical attacks in a year or three in 4-6 months with an elevated acute phase response between attacks," and with the new EULAR recommendations, at least 1 attack/month in a 6-month period with full adherence to colchicine treatment $[4,13]$. Another finding is that a number of patients, especially children, received doses of colchicine higher than that recommended and experienced digestive symptoms of intolerance, which could be considered not strictly synonymous with resistance to treatment. Colchicine is mainly absorbed from jejunal and ileal mucosa and mainly eliminated via biliary excretion (10-20\% renal excretion). Anorexia, nausea, diarrhoea, and increased liver enzyme activity are the most common signs of intolerance; these can be overcome in part by twice-daily divided doses, but there are also probably individual differences regarding this issue [14]. Of note, severe fatal intoxications have been reported with concomitant use of clarithromycin and other drugs using the same cytochrome P450 metabolic pathways, which can lead to colchicine accumulation apart from situations of overdose. Alimentary factors may also be involved, and the concomitant intake of grapefruit juice or plants (St. John's wort) may also increase colchicine toxicity. Another major issue raised by our study and others is the very low full adherence to daily colchicine treatment (40\%), even lower than previously reported (60\%), which can only be partially explained by digestive intolerance [14]. Indeed, paediatric patients received higher doses than adults and had more side effects but were more fully adherent than adults ( $48 \%$ vs $22 \%$ ). The international recommendations distinguish low adherence from resistance to colchicine treatment $[4,13]$, but the practical way to improve the management of this critical issue needs to be determined. New findings have shown that psychological "stress" is sensed by the innate immune system in the brain via the ATP/P2X7R-NLRP3 inflammasome cascade; reversing the activation of this pathway in mice blocked the release of IL-1 $\beta$ (1-3 days after infusion) and produced antidepressant and anxiolytic behavioural effects in nonstressed mice [15]. Many of our patients could benefit from biological treatments that were exclusively anti-IL-1 drugs. Although not within the scope of this study, these treatments gave good response for FMF-related musculoskeletal symptoms and secondary amyloidosis. According to international recommendations, all patients were prescribed daily colchicine, when still possible. 


\section{Conclusion}

Despite our retrospective study with some missing data and possible recall bias, it brings important insights into factors affecting inadequate response to colchicine treatment for FMF. Besides the severity of the disease itself, low adherence to treatment is of major importance and remains a challenge for all physicians. Searching for possible causes of digestive intolerance and promoting patient education to reinforce stress control and encourage adherence to treatment may decrease the need for expensive alternative treatments.

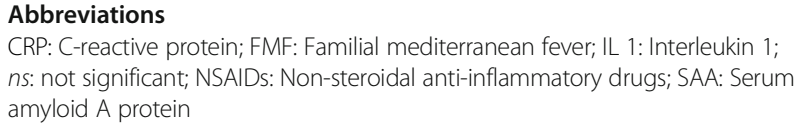

\section{Acknowledgements}

We thank Laura Smales (http://www.biomedediting.com), for editing the manuscript for English language.

\section{Funding \\ None.}

\section{Availability of data and materials}

Please contact author for data requests.

\section{Authors' contributions}

IKP and AC have conceived the study, contributed to data acquisition and statistical analyses, and written the manuscript. IKP, SGL, VH, EH, GG, AF, PQ, LRS managed patients, and contributed to data acquisition and manuscript preparation. All authors read and approved the final manuscript."

\section{Competing interests}

Alice Corsia, Sophie Georgin Lavialle, Gilles Grateau and Albert Faye, and have no conflicts of interest to declare. Véronique Hentgen has received consulting and meeting fees from Novartis. Pierre Quartier has received consultancy, speaker's fees or honoraria from Novartis or Sobi; Eric Hachulla has received consultancy, speaker's fees or honoraria from Novartis and Sobi. Linda Rossi-Semerano has received congressional funding from: Abbvie, Novartis, SOBI Biovitrum, Pfizer, Roche Chugai. Isabelle Koné-Paut has received consultancy fees and congressional funding from Novartis, Sobi, Pfizer and Abbvie.

\section{Consent for publication}

According to our national regulations, patient's consent was not required for this type of non interventional study.

\section{Ethics approval and consent to participate}

According the national regulations, it is not required for this type of study.

\section{Collaborators}

Nicolas Schleinitz, Department of Internal Medicine, Conception Hospital, APHP, University of Aix Marseille, Marseille, France, Yvan Jamilloux, Department of Internal Medicine, De la Croix Rousse Hospital, Lyon, France, Gilles Hayem, Department of Internal Medicine and Infectious Diseases, Ambroise Paré Hospital, Paris, France, Ulrich Meinzer, General Paediatrics, Robert Debré Hospital, AP-HP, University of Paris, France

\section{Publisher's Note}

Springer Nature remains neutral with regard to jurisdictional claims in published maps and institutional affiliations.

\section{Author details}

'Department of Paediatrics Rheumatology, CEREMAl, Bicêtre Hospital, AP-HP, University of Paris SUD, Le Kremlin-Bicêtre, France. ${ }^{2}$ Internal medicine, CERAIF, Tenon hospital, AP-HP, University of Paris EsT, Paris, France.

${ }^{3}$ Paediatrics, CEREMAI, Mignot Hospital, Versailles-Le Chainay, France. ${ }^{4}$ Internal medicine, Huriez hospital, University of Lille, Lille, France. ${ }^{5}$ General Paediatrics, Robert Debré Hospital, AP-HP, University of Paris, Paris, France. ${ }^{6}$ Paediatric rheumatology and immunology, CERHUMIP, Necker Hospital, University of Paris, Paris, France.

Received: 4 December 2016 Accepted: 8 March 2017

Published online: 16 March 2017

References

1. Ben-Chetrit E, Touitou I. Familial Mediterranean fever in the world. Arthritis Rheum. 2009;61:1447-53. doi:10.1002/art.24458.

2. Zemer D, Revach M, Pras M, Modan B, Schor S, Sohar E, et al. A controlled trial of colchicine in preventing attacks of familial Mediterranean fever. N Engl J Med. 1974;291:932-4. doi:10.1056/NEJM197410312911803.

3. Kallinich T, Haffner D, Niehues T, Huss K, Lainka E, Neudorf U, et al. Colchicine use in children and adolescents with familial Mediterranean fever: literature review and consensus statement. Pediatrics. 2007;119:e47483. doi:10.1542/peds.2006-1434.

4. Hentgen V, Grateau G, Kone-Paut I, Livneh A, Padeh S, Rozenbaum M, et al. Evidence-based recommendations for the practical management of Familial Mediterranean Fever. Semin Arthritis Rheum. 2013;43:387-91. doi:10.1016/j. semarthrit.2013.04.011

5. Ben-Chetrit E, Ozdogan $H$. Non-response to colchicine in FMF-definition, causes and suggested solutions. Clin Exp Rheumatol. 2008:26:S49-51.

6. Meinzer U, Quartier P, Alexandra J-F, Hentgen V, Retornaz F, Koné-Paut I. Interleukin-1 targeting drugs in familial Mediterranean fever: a case series and a review of the literature. Semin Arthritis Rheum. 2011;41:265-71. doi: 10.1016/j.semarthrit.2010.11.003.

7. Gül A, Ozdogan H, Erer B, Ugurlu S, Kasapcopur O, Davis N, et al. Efficacy and safety of canakinumab in adolescents and adults with colchicineresistant familial Mediterranean fever. Arthritis Res Ther. 2015;17:243. doi:10. 1186/s13075-015-0765-4

8. Mor A, Shinar Y, Zaks N, Langevitz P, Chetrit A, Shtrasburg S, et al. Evaluation of disease severity in familial Mediterranean fever. Semin Arthritis Rheum. 2005:35:57-64. doi:10.1016/j.semarthrit.2005.02.002

9. Lidar M, Scherrmann J-M, Shinar Y, Chetrit A, Niel E, Gershoni-Baruch R, et al. Colchicine nonresponsiveness in familial Mediterranean fever: clinical, genetic, pharmacokinetic, and socioeconomic characterization. Semin Arthritis Rheum. 2004:33:273-82.

10. Özçakar ZB, Elhan AH, Yalçınkaya F. Can colchicine response be predicted in familial Mediterranean fever patients? Rheumatol Oxf Engl. 2014;53:1767-72. doi:10.1093/rheumatology/keu138.

11. Hashkes PJ, Spalding SJ, Hajj-Ali R, Giannini EH, Johnson A, Barron KS, et al. The effect of rilonacept versus placebo on health-related quality of life in patients with poorly controlled familial Mediterranean fever. BioMed Res Int. 2014:2014:854842. doi:10.1155/2014/854842.

12. Akar S, Soysal O, Balci A, Solmaz D, Gerdan V, Onen F, et al. High prevalence of spondyloarthritis and ankylosing spondylitis among familial Mediterranean fever patients and their first-degree relatives: further evidence for the connection. Arthritis Res Ther. 2013:15:R21. doi:10.1186/ar4154.

13. Ozen S, Demirkaya E, Erer B, Livneh A, Ben-Chetrit E, Giancane G, et al. EULAR recommendations for the management of familial Mediterranean fever. Ann Rheum Dis. 2016;75:644-51. doi:10.1136/annrheumdis-2015-208690.

14. Ben-Chetrit E, Aamar S. About colchicine compliance, resistance and virulence. Clin Exp Rheumatol. 2009:27:S1-3.

15. Iwata M, Ota KT, Li X-Y, Sakaue F, Li N, Dutheil S, et al. Psychological stress activates the inflammasome via release of adenosine triphosphate and stimulation of the purinergic type 2X7 receptor. Biol Psychiatry. 2016;80:12-22. doi:10.1016/j.biopsych.2015.11.026. 